\title{
A Note on Solving Discretely-Constrained Nash-Cournot Games via Complementarity
}

\author{
Dimitri J. Papageorgiou*, Francisco Trespalacios, Stuart Harwood \\ Corporate Strategic Research \\ ExxonMobil Research and Engineering Company \\ 1545 Route 22 East, Annandale, NJ 08801 USA \\ \{dimitri.j.papageorgiou,francisco.trespalacios,stuart.harwood\}@exxonmobil.com \\ ${ }^{*}$ Corresponding author
}

March 4, 2020

\begin{abstract}
Discretely-constrained Nash-Cournot games have attracted attention as they arise in various competitive energy production settings in which players must make one or more discrete decisions. Gabriel et al. [2] claim that the set of equilibria to a discretely-constrained Nash-Cournot game coincides with the set of solutions to a corresponding discretely-constrained mixed complementarity problem. We show that this claim is false.
\end{abstract}

Keywords: Complementarity; Equilibria; Integrality; Nash-Cournot games; Relaxation.

\section{Introduction}

A Nash-Cournot game is a game-theoretical framework of imperfect competition in which multiple producers/players compete to optimize their individual objective functions, which also depend on other players' production decisions. Traditional (i.e., purely continuous) Nash-Cournot problems have been extensively studied and it is well known that they can be expressed either as nonlinear complementarity or variational inequality problems [1]. Discretely-constrained Nash-Cournot (DC-NC) games arise when a subset of a player's decisions are required to be discrete, for example, when a player must make a binary on/off decision. Gabriel et al. [2] approached discretely-constrained Nash-Cournot games by framing the problem as a discretely-constrained mixed complementarity problem (DC-MCP).

We consider the same set up and, to the extent possible, the same notation as Gabriel et al. [2]. There are $N$ players indexed by $p \in \mathcal{P}=\{1, \ldots, N\}$. Player $p$ optimizes her cost function $f_{p}: \mathbb{R}^{n} \mapsto \mathbb{R}$ that depends on her decision vector $\mathbf{x}_{p} \in \mathbb{R}^{n_{p}}$ and the vector $\mathbf{x}_{-p}=\left(\mathbf{x}_{1}, \ldots, \mathbf{x}_{p-1}, \mathbf{x}_{p+1}, \ldots, \mathbf{x}_{N}\right)$ denoting the decisions of all other players besides player $p$. Here, $n=\sum_{p \in \mathcal{P}} n_{p}$. Specifically, we assume that player $p$ 
solves the following discretely-constrained optimization problem parameterized by $\mathbf{x}_{-p}$ :

$$
\begin{array}{clll}
f_{p}^{*}\left(\mathbf{x}_{-p}\right)=\min _{\mathbf{x}_{p}} & f_{p}\left(\mathbf{x}_{p}, \mathbf{x}_{-p}\right) & \text { [dual vars] } & \\
\text { s.t. } & g_{p j}\left(\mathbf{x}_{p}\right) \leq 0 & {\left[\lambda_{p j} \geq 0\right]} & \forall j \in \mathcal{I}_{p} \\
& h_{p k}\left(\mathbf{x}_{p}\right)=0 & {\left[\gamma_{p k} \in \mathbb{R}\right]} & \forall k \in \mathcal{E}_{p} \\
& \mathbf{x}_{p} \geq \mathbf{0} & & \\
& x_{p r} \in \mathbb{Z}_{+} & & \forall r \in \mathcal{D}_{p},
\end{array}
$$

where $\mathcal{I}_{p}, \mathcal{E}_{p}$, and $\mathcal{D}_{p}$ denote the set of inequalities, equalities, and integer variables for player $p \in \mathcal{P}$. Let $\mathcal{X}_{p}=\left\{\mathbf{x}_{p} \in \mathbb{R}^{n_{p}}:(1 \mathrm{~b}),(1 \mathrm{c}),(1 \mathrm{~d}),(1 \mathrm{e})\right\}$ denote the discretely-constrained feasible region for player $p \in \mathcal{P}$ and let $\mathcal{C}_{p}=\left\{\mathbf{x}_{p} \in \mathbb{R}^{n_{p}}:(1 \mathrm{~b}),(1 \mathrm{c}),(1 \mathrm{~d})\right\}$ denote the continuous relaxation of $\mathcal{X}_{p}$. A vector $\hat{\mathbf{x}}$ is called a Nash equilibrium of this DC-NC game if $\hat{\mathbf{x}}_{p} \in \mathcal{X}_{p}$ for all $p \in \mathcal{P}$ and

$$
f_{p}\left(\hat{\mathbf{x}}_{p}, \hat{\mathbf{x}}_{-p}\right) \leq f_{p}\left(\mathbf{x}_{p}, \hat{\mathbf{x}}_{-p}\right), \quad \forall p \in \mathcal{P}, \mathbf{x}_{p} \in \mathcal{X}_{p} .
$$

Gabriel et al. [2] approach convex DC-NC games, i.e., games in which the continuous relaxation of each player's optimization problem is a convex optimization problem, by applying the following four-step procedure: 1) relax the integrality constraints for each player; 2) write the KKT conditions for each player; 3) re-impose the integrality constraints; 4) solve the resulting DC-MCP. More concretely, since KKT conditions are neither necessary nor sufficient for a discrete optimization problem, Gabriel et al. [2] attempt to find the set of Nash equilibria to (2) by appealing to the continuous relaxation of each player's parametric optimization problem:

$$
\min \left\{f_{p}\left(\mathbf{x}_{p}, \mathbf{x}_{-p}\right): \mathbf{x}_{p} \in \mathcal{C}_{p}\right\}
$$

Assume that the functions $f_{p}\left(\cdot, \mathbf{x}_{-p}\right)$ are convex and a constraint qualification for the continuous relaxation $\mathcal{C}_{p}$ holds. Then, the KKT conditions for player $p$ 's relaxed problem (3) are to find $\mathbf{x}_{p} \in \mathbb{R}^{n_{p}}, \lambda_{p} \in \mathbb{R}^{\left|\mathcal{I}_{p}\right|}$, $\gamma_{p} \in \mathbb{R}^{\left|\mathcal{E}_{p}\right|}$ such that

$$
\begin{aligned}
& \mathbf{0} \leq \nabla_{\mathbf{x}_{p}} f_{p}\left(\mathbf{x}_{p}, \mathbf{x}_{-p}\right)+\sum_{j \in \mathcal{I}_{p}} \lambda_{p j} \nabla g_{p j}\left(\mathbf{x}_{p}\right)+\sum_{k \in \mathcal{E}_{p}} \gamma_{p k} \nabla h_{p k}\left(\mathbf{x}_{p}\right) \perp \mathbf{x}_{p} \geq \mathbf{0} \\
& 0 \leq-g_{p j}\left(\mathbf{x}_{p}, \mathbf{x}_{-p}\right) \perp \lambda_{p j} \geq 0 \forall j \in \mathcal{I}_{p} \\
& h_{p k}\left(\mathbf{x}_{p}, \mathbf{x}_{-p}\right)=0, \gamma_{p k} \in \mathbb{R} \forall k \in \mathcal{E}_{p}
\end{aligned}
$$

Gabriel et al. [2] (p.313) then write:

"An interesting question is whether the set of $\mathbf{x}_{p}$ that solves (4), but with the discrete restrictions for $x_{p r} \in \mathbb{Z}_{+}$for $r \in \mathcal{D}_{p}$, corresponds to the solution set of the original problem (2). The next result shows that this correspondence is correct."

Theorem 1 (Theorem 3 in [2]) Let $\mathcal{S}^{\text {DC-Nash }}$ be the set of solutions to the discretely-constrained NashCournot game (2) and $\mathcal{S}^{D C-M C P}$ be the set of solutions to (4) for which $x_{p r} \in \mathbb{Z}_{+}$for $r \in \mathcal{D}_{p}$. Then, $\mathcal{S}^{D C-N a s h}=\mathcal{S}^{D C-M C P}$.

\section{Counterexamples}

We now provide two simple discretely-constrained Nash-Cournot duopoly games (i.e., $\mathcal{P}=\{1,2\}$ ) for which one or more equilibria exist to (2), but the complementarity conditions coupled with integrality restrictions 
are either 1) empty, or 2) non-empty, but a strict subset of the true set of equilibria. In both examples, because each player controls a single decision variable, we index player $p$ 's decision variable as $x_{p}$ rather than $x_{p 1}$.

\section{1 "Linear" players with weak continuous relaxations}

Consider the simple Nash-Cournot duopoly game with the following symmetric payoff matrix:

\begin{tabular}{c|cc} 
& $x_{2}=0$ & $x_{2}=1$ \\
\hline$x_{1}=0$ & 0 & -1 \\
$x_{1}=1$ & -1 & -2
\end{tabular}

Here each player can take a discrete (binary) action with the unique equilibrium being $x_{1}=x_{2}=1$, i.e., each player chooses action 1 for a (minimum) payoff of -2 , which is obviously a dominant strategy for each player. We now translate this DC-NC game into an optimization framework. Suppose player $p \in\{1,2\}$ solves the following problem:

$$
f_{p}^{*}\left(x_{-p}\right)=\min \left\{-x_{p}-x_{-p}: x_{p} \in[0,1+\epsilon] \cap \mathbb{Z}\right\}
$$

where $\epsilon>0$ and $\mathbb{Z}$ is the set of integers. The corresponding KKT optimality conditions are

$$
\begin{array}{lll}
0 \leq \lambda_{p}-1 & \perp x_{p} \geq 0 & \forall p \\
0 \leq 1+\epsilon-x_{p} \perp \lambda_{p} \geq 0 & \forall p
\end{array}
$$

We now plug in the unique equilibrium solution $x_{1}=x_{2}=1$. Complementarity conditions (6a) imply that $\lambda_{p}=1$, while conditions $(6 \mathrm{~b})$ imply that $\lambda_{p}=0$. This contradiction reveals that the unique equilibrium solution $x_{1}=x_{2}=1$ is not in $\mathcal{S}^{\text {DC-MCP }}$, i.e. $\emptyset=\mathcal{S}^{\text {DC-MCP }} \subset \mathcal{S}^{\text {DC-Nash }} \neq \emptyset$.

\section{2 "Quadratic" players with tight continuous relaxations}

In this example, the continuous relaxation for each player is tight. Consider the payoff matrix

$$
\begin{array}{c|cc} 
& x_{2}=0 & x_{2}=1 \\
\hline x_{1}=0 & (0,0) & (9,9) \\
x_{1}=1 & (4,4) & (1,1-\delta)
\end{array}
$$

For $\delta>-3$, there are two equilibria in pure strategies: $\left(x_{1}, x_{2}\right)=(0,0)$ and $\left(x_{1}, x_{2}\right)=(1,1)$.

This corresponds to player 1 solving the following convex quadratic problem (as a function of $x_{2}$ ):

$$
\begin{aligned}
f_{1}^{*}\left(x_{2}\right)=\min _{x_{1}} & \left(2 x_{1}-3 x_{2}\right)^{2} \\
\text { s.t. } & x_{1}-1 \leq 0 \\
& -x_{1} \leq 0
\end{aligned}
$$

Meanwhile, player 2 solves a similar convex quadratic problem (as a function of $x_{1}$ ):

$$
\begin{aligned}
f_{2}^{*}\left(x_{1}\right)=\min _{x_{2}} & \left(2 x_{1}-3 x_{2}\right)^{2}-\delta x_{1} x_{2} \\
\text { s.t. } & x_{2}-1 \leq 0 \\
& -x_{2} \leq 0
\end{aligned}
$$


Note that $f_{p}\left(\cdot, \mathbf{x}_{-p}\right)$ are convex functions and a constraint qualification holds.

The KKT conditions (4) become

$$
\begin{array}{lrl}
0 \leq 4\left(2 x_{1}-3 x_{2}\right)+\lambda_{1} & \perp x_{1} \geq 0 \\
0 \leq-6\left(2 x_{1}-3 x_{2}\right)-\delta x_{1}+\lambda_{2} & \perp x_{2} \geq 0 \\
0 \leq 1-x_{p} & \perp \lambda_{p} \geq 0 \quad \forall p
\end{array}
$$

Assume $\delta>-3$. It is straightforward to verify that $x_{p}=\lambda_{p}=0$ for all $p$ satisfy the complementarity conditions (9). The situation is different for $\left(x_{1}, x_{2}\right)=(1,1)$. Condition (9a) implies that $\lambda_{1}=4$, while condition (9c) implies that $\lambda_{p} \geq 0$ for all $p$. However, condition (9b) implies that $\lambda_{2}=-6+\delta$. Thus, for $\delta \in(-3,6)$, the complementarity approach fails to recognize $\left(x_{1}, x_{2}\right)=(1,1)$ as an equilibrium. It it tempting to argue that when $\delta \in(-3,1]$, this omission is not a concern because $\left(x_{1}, x_{2}\right)=(0,0)$ is the preferred equilibrium (i.e., the global minimizer for both players). However, for $\delta>1$, player 2 's global minimizer is $\left(x_{1}, x_{2}\right)=(1,1)$ with a payoff of $1-\delta$ and, for $\delta \in(1,6)$, the complementarity approach does not "see" this solution as an equilibrium. In short, this example shows that, not only can the complementarity approach fail to find all equilibria to a DC-NC game, it is not guaranteed to find global optima for each player when it does return an equilibrium.

Note that one can obtain a similar result (counterexample) by replacing the $L 2$ term $\left(2 x_{1}-3 x_{2}\right)^{2}$ with the $L 1$ term $\left|2 x_{1}-3 x_{2}\right|$ so that each player solves a linear optimization problem instead of a convex quadratic one.

\section{Resolution}

For completeness, the correct version of Theorem 3 in Gabriel et al. is

Theorem 2 Let $\mathcal{S}^{D C-N a s h}$ be the set of solutions to the discretely-constrained Nash-Cournot game (2) and $\mathcal{S}^{D C-M C P}$ be the set of solutions to (4) for which $x_{p r} \in \mathbb{Z}_{+}$for $r \in \mathcal{D}_{p}$. Then, $\mathcal{S}^{D C-M C P} \subseteq \mathcal{S}^{D C-N a s h}$ and there exist cases when $\mathcal{S}^{D C-M C P} \subsetneq \mathcal{S}^{D C-N a s h}$.

Finally, note that the heuristic proposed by Gabriel et al. to solve the DC-NC game (2) is still valid.

\section{References}

[1] F. Facchinei and J.-S. Pang. Finite-dimensional variational inequalities and complementarity problems. Springer Science \& Business Media, 2007.

[2] S. A. Gabriel, S. A. Siddiqui, A. J. Conejo, and C. Ruiz. Solving discretely-constrained Nash-Cournot games with an application to power markets. Networks and Spatial Economics, 13(3):307-326, Sep 2013. 